\title{
Plicatura gástrica laparoscópica, un nuevo procedimiento bariátrico: a propósito de un caso clínico*
}

\author{
Drs. JUAN EDUARDO CONTRERAS P. ${ }^{1}$, DIVA VILLAO M. ${ }^{1}$, JORGE BRAVO L. ${ }^{1}$, \\ JULIO NÚÑEZ V. ${ }^{1}$, JUAN PABLO CAMACHO M. ${ }^{1}$, GUSTAVO CZWIKLITZER S. ${ }^{1}$, \\ CARLOS DÍAZ-VALDÉS A. ${ }^{1}$, ISMAEL COURT R. ${ }^{1}$ \\ 1 Hospital del Salvador, Servicio y Departamento de Cirugía, Sede Oriente, Facultad de Medicina, \\ Universidad de Chile, Santiago, Chile.
}

\begin{abstract}
Laparoscopic gastric plication, a new bariatric procedure: report of one case

Bariatric surgery is an effective treatment for morbid obesity. Various techniques have been developed with different outcomes and complications. A new technique with promising results recently described in the literature: the gastric plication, which would not have the complications of using mechanical suture and would imply a reduction of costs. We report the first case in Chile under this technique.
\end{abstract}

Key words: Morbid obesity, bariatric surgery, gastroplasty, gastric plication.

\section{Resumen}

La cirugía bariátrica es un tratamiento efectivo para la obesidad mórbida. Diversas técnicas se han desarrollado con diferentes resultados y complicaciones. Una nueva técnica con resultados prometedores se ha descrito recientemente en la literatura: la plicatura gástrica, la cual no tendría las complicaciones del uso de sutura mecánica e implicaría una reducción de los costos. Presentamos el caso clínico del primer paciente en Chile sometido a esta técnica.

Palabras clave: Obesidad mórbida, cirugía bariátrica, gastroplastía, plicatura gástrica.

\section{Introducción}

La obesidad es la epidemia del siglo XXI. Según la organización mundial de la salud, Arabia Saudita y Estados Unidos tienen 72,5\% y 66,9\% de su población con un índice de masa corporal (IMC) mayor o igual a $25 \mathrm{~kg} / \mathrm{mt}^{2}$, respectivamente ${ }^{1}$. En nuestro país este porcentaje aumentó recientemente a un $67 \%^{2}$. La obesidad está ligada a comorbilidades tales como hipertensión arterial, diabetes, dislipidemia, apnea del sueño, enfermedad coronaria e infartos cerebrales ${ }^{3,4}$. La cirugía bariátrica reduce la ingesta calórica modificando la anatomía del tracto gastrointestinal. Éstas pueden ser cla-

*Recibido el 12 de enero de 2011 y aceptado para publicación el 4 de mayo de 2011.

Correspondencia: Dr. Juan E. Contreras P.

El Salvador \#364, Santiago, Chile.

juaneduardocontreras@gmail.com 
sificadas como restrictivas, malabsortivas y sus combinaciones ${ }^{4}$. Estudios de la última década han agregado el concepto de cirugía metabólica, evidenciando posteriormente cambios en la regulación de incretinas, y una mejoría e incluso desaparición de comorbilidades $^{5,6}$.

Uno de los procedimientos que más se realiza actualmente es la gastrectomía vertical tubular, sin embargo, los costos y complicaciones como filtraciones o estenosis han incentivado la búsqueda de otras alternativas para este procedimiento. Así una nueva técnica llamada plicatura gástrica ha sido reportada recientemente ${ }^{7-12}$.

El objetivo de este trabajo es mostrar la técnica quirúrgica del primer caso realizado en Chile.

\section{Caso clínico}

Paciente de 34 años de edad, femenino, presenta obesidad con infructuoso tratamiento en atención primaria. Comorbilidades asociadas: hipertensión arterial, dislipidemia, apnea del sueño, resistencia a la insulina, esteatosis hepática, várices, tabaquismo activo y depresión en tratamiento. Es derivada a la unidad de cirugía bariátrica del Hospital del Salvador. En julio de 2009, informe realizado por nutricionista constata un peso inicial de $111 \mathrm{~kg}$ e IMC de 38,4 kg/mt².

Se decide disminuir el aporte calórico a 1.400 Kcalorías/día promedio. En diciembre de 2009, se objetiva peso de 106,9 kg e IMC de 36,9 kg/mt. Paciente no incorporó actividad física diariamente por una tendinitis en rodilla, en tratamiento kinesiológico 2 veces por semana.

Debido al fallido tratamiento médico, se decide programar para cirugía, siendo ingresada al protocolo del Hospital del Salvador para plicatura gástrica.

Este protocolo fue rigurosamente evaluado y aprobado por el comité de ética, y el servicio de cirugía del Hospital del Salvador, sustentado en la bibliografía publicada.

Se confeccionó un consentimiento informado especial destacando que se trata de una operación dentro de un protocolo de investigación clínica.

Exámenes pre-operatorios destacan: glicemia basal $84 \mathrm{mg} / \mathrm{dl}$, glicemia postcarga $126 \mathrm{mg} / \mathrm{dl}$, insulina basal 23,7 uU/ml, triglicéridos $262 \mathrm{mg} / \mathrm{dl}$, colesterol total $162 \mathrm{mg} / \mathrm{dl}$, colesterol HDL $27 \mathrm{mg} / \mathrm{dl}$, hematocrito 41,8\%, TSH 0,66 uUI/ml y T3 1,93 ng/ml. Endoscopía digestiva alta: sin hallazgos patológicos, con test de ureasa positivo tratado. Radiografía tórax y espirometría en límites normales. Ecografía: hígado con infiltración grasa, resto normal. Previo a cirugía la paciente es sometida durante 1 semana a un régimen líquido.

\section{Técnica quirúrgica}

Bajo anestesia general se coloca paciente en decúbito dorsal con técnica francesa. Colocación de aguja de Veress en hipocondrio izquierdo, posteriormente neumoperitoneo a 15 mmHg. Inserción de trocares según técnica estándar para gastrectomía tubular vertical laparoscópica: primer trocar de 15 $\mathrm{mm}$ a $15 \mathrm{~cm}$ de apéndice xifoides, accediendo a través de Visiport ${ }^{\circledR}$ y por el que se introduce cámara de $30^{\circ}$. Segundo trocar de $12 \mathrm{~mm}$, se accede también a través de Visiport ${ }^{\circledR}$, a aproximadamente $8 \mathrm{~cm}$ del anterior, en dirección superior izquierda del paciente, el cual funciona como principal puerto de trabajo y segunda alternativa para cámara. Luego 3 trocares de $5 \mathrm{~mm}$ : el primero en el flanco derecho del paciente a $8 \mathrm{~cm}$ superior derecha del primer trocar colocado, para la mano izquierda del cirujano; el segundo bajo el apéndice xifoides, para la separación del hígado por parte del asistente; y el tercero a nivel de la línea axilar, para el asistente (Figura 1).

Con bisturí Harmónico ${ }^{\circledR}$ se esqueletiza estómago desde $6 \mathrm{~cm}$ del píloro, hasta el ángulo de His con línea de sección a $2 \mathrm{~cm}$ por fuera del borde la curvatura mayor, para evitar dañar el borde gástrico. Bajo calibración con sonda número 42 y apoyo endoscópico simultáneo, se procede a una primera invaginación de la curvatura mayor gástrica con puntos separados de seda 2/0. Luego una segunda invaginación con punto continuo de prolene 2/0 (Figuras 2, 3, 4 y 5). Se comprueba hemostasia, recuento de gasas y buen paso de endoscopio a distal. Se electrofulguran los puertos bajo visión laparoscópica y se realiza cierre de pared por planos.

Durante el período postoperatorio, en las primeras 24 horas, paciente evolucionó con náuseas y vómitos que cedieron precozmente.

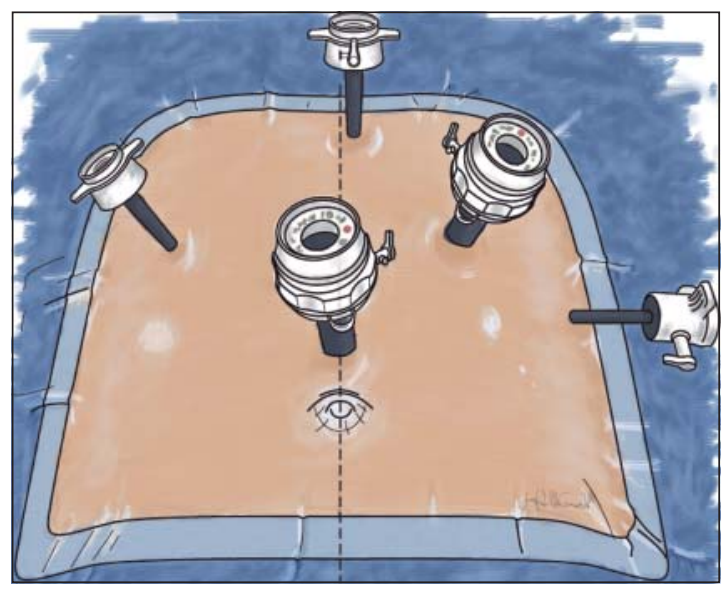

Figura 1. Colocación de los puertos según técnica clásica para gastrectomía vertical laparoscópica. 


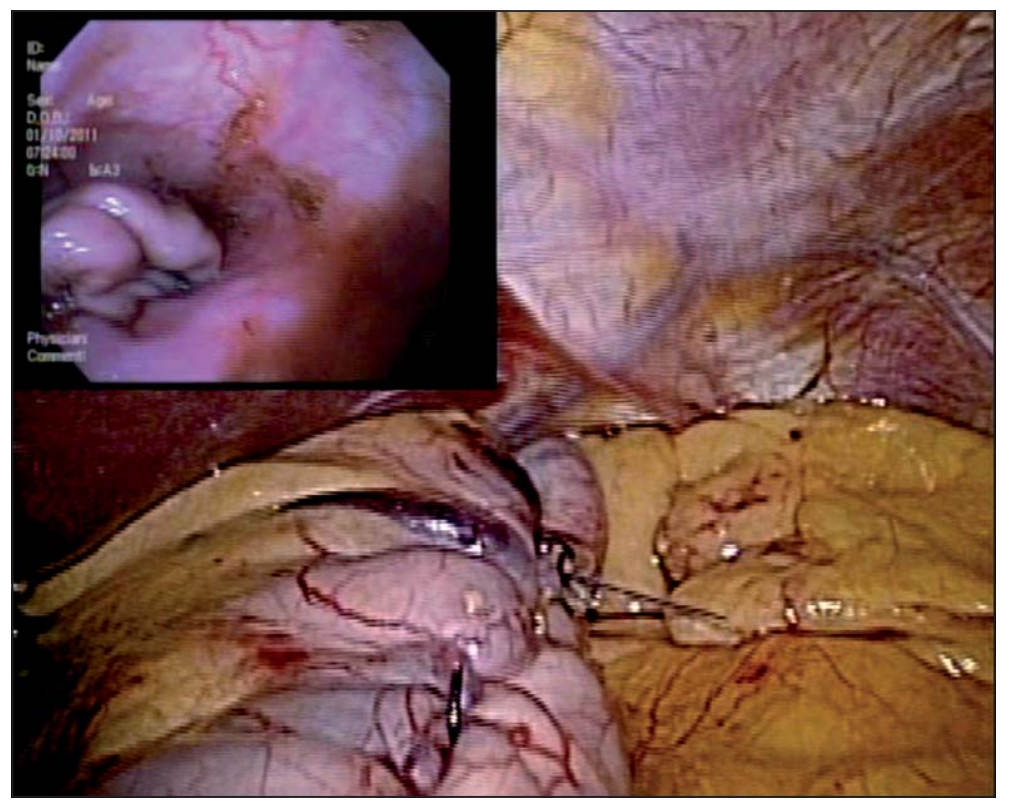

Figura 2. Visualización de plicatura gástrica terminada con imagen endoscópica simultánea. Se observa en la imagen endoscópica pliegue intraluminal producido por la invaginación.

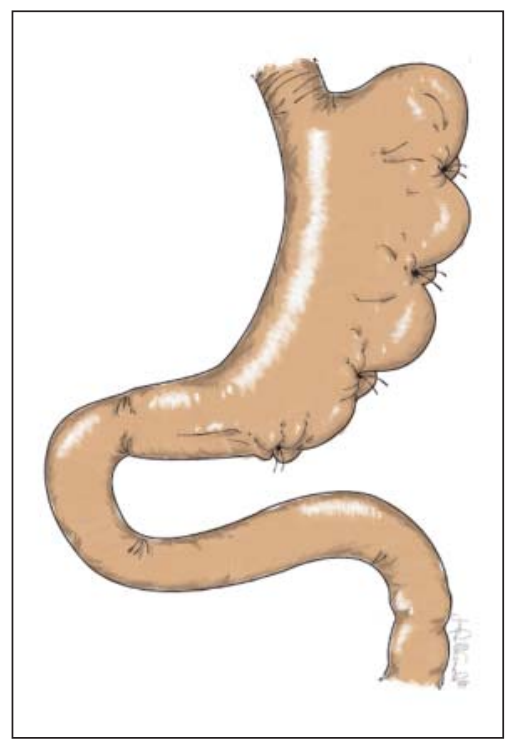

Figura 3. Esquema de plicatura gástrica laparoscópica luego de colocación de puntos separados de seda.

La paciente fue dada de alta a las 48 horas ingiriendo líquidos. Se recomendó dieta líquida por 15 días, luego papilla por 2 semanas.

Se realizó tránsito intestinal durante la segunda semana, evidenciando paso de medio de contraste hasta duodeno con facilidad, sin filtraciones (Figura 6).

\section{Discusión}

La obesidad es uno de los grandes problemas a resolver durante las próximas décadas, conlleva

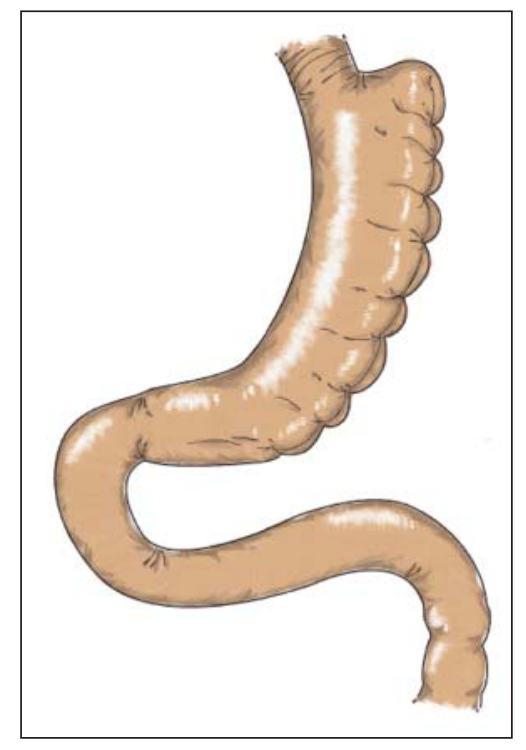

Figura 4. Esquema de plicatura gástrica laparoscópica luego de sutura contínua de prolene.

comorbilidades que aumentan la morbi-mortalidad de la población, es en este punto en donde radica la importancia de tratarla ${ }^{1-4}$.

Las fallas en dietas, ejercicio y terapia médica, además del aumento de los pacientes con obesidad extrema han aumentado el número de cirugías bariátricas $^{3,13}$. Existen diferentes técnicas, las que pueden dividirse según su mecanismo de acción en: restrictivas, malabsortivas y sus combinaciones ${ }^{4,5}$. Comorbilidades tales como diabetes, hipertensión arterial, dislipidemia, entre otras, presentan mejoría 


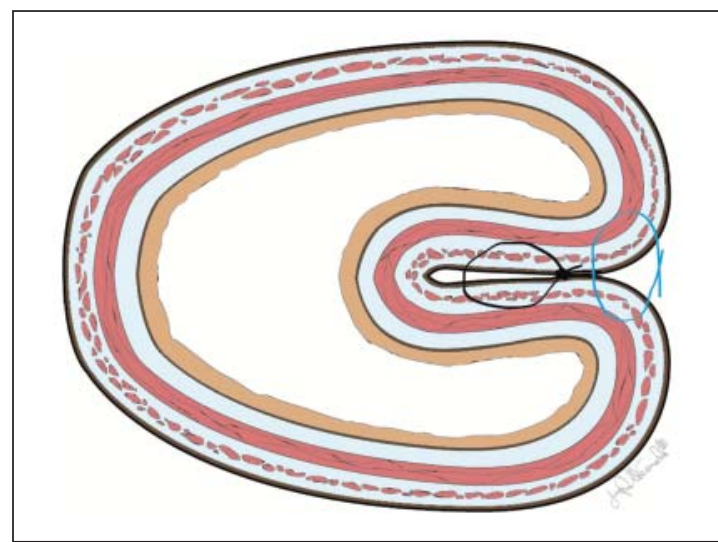

Figura 5. Esquema de corte transversal de plicatura gástrica laparoscópica terminada. En negro puntos separados de seda y en azul punto contínuo de prolene.

y/o remiten en un gran porcentaje luego del procedimiento; teniendo un rol importante las incretinas ${ }^{5,14}$. Incluso se ha demostrado una disminución de la mortalidad de los pacientes sometidos a cirugía con respecto al grupo control ${ }^{15}$. Debido a que es un procedimiento relativamente joven, sus indicaciones y técnicas han variado bastante en los últimos 20 años, y aun quedan mecanismos fisiopatológicos por descubrir ${ }^{5,14}$.

Los costos y complicaciones de los actuales procedimientos bariátricos han incentivado la búsqueda de otras alternativas en cirugía ${ }^{7,9,11}$.

En el año 2005, Neumayer, publicó un artículo en el cual logra objetivar una baja de peso estadísticamente significativa en pacientes sometidos a fundoplicatura de Nissen ${ }^{16}$. Este trabajo fue utilizado como referencia por un grupo brasileño a cargo de Fusco. Este estudio se realizó en ratas, dividiéndolas en 3 grupos: uno con plicatura de curvatura mayor, otro de manipulación de vísceras por 15 minutos, y otro control. Se evidencia una significativa baja de peso en el primer grupo, en comparación a los otros dos$^{7}$. Otro estudio, también en ratas, el mismo grupo logra demostrar que la plicatura de la curvatura mayor gástrica es más efectiva en la reducción de peso que la plicatura de la pared anterior ${ }^{8}$.

En base a estos resultados no tardan en aparecer los artículos de las primeras series en humanos. En el año 2007, Talebpour publica 100 casos de pacientes sometidos a plicatura gástrica, logrando un $60 \%$ de reducción del exceso de peso al año ${ }^{10}$. Durante el 2008 Sales, presenta, en la Revista Colombiana de Cirugía, una reducción del exceso de peso de 69,6\% luego del procedimiento, ahora bien, no menciona el momento del control ${ }^{9}$.

Recientemente, en el año 2010, el grupo de Almi-

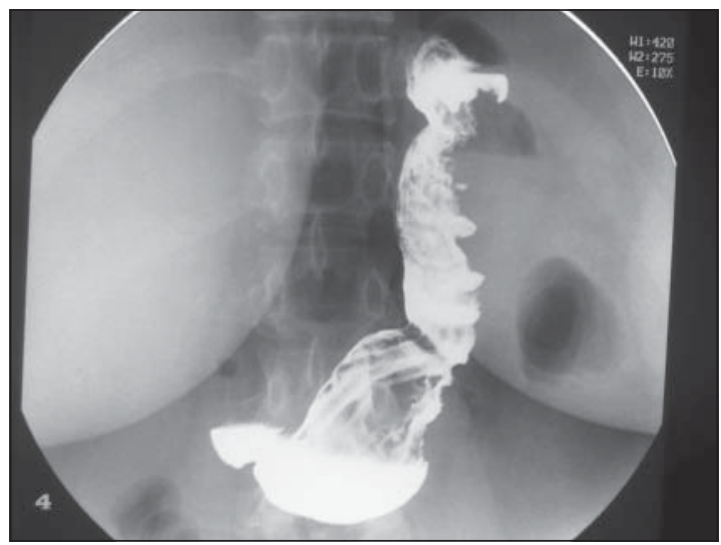

Figura 6. Tránsito intestinal realizado durante la segunda semana de postoperatorio. Se observa una marcada reducción del lumen gástrico.

no Ramos publica una serie de 42 pacientes, demostrando una reducción promedio del exceso de peso de $62 \%$ en 9 casos a los 18 meses. Estos resultados preliminares serían similares a los obtenidos a través de una gastrectomía vertical laparoscópica ${ }^{11}$. Finalmente, el grupo de la Cleveland Clinic, presenta una pequeña serie de 15 pacientes, la cual fue dividida en 2 grupos: en 9 pacientes se realizó una plicatura gástrica anterior y en 6 una plicatura gástrica de la curvatura mayor. Completaron el año de seguimiento 6 y 5 pacientes, con una baja de peso de $23,3 \% \pm$ $24,8 \%$ y $53,4 \% \pm 22,7 \%$, respectivamente. Con estos resultados se objetivó una mayor baja de peso con la plicatura de la curvatura mayor ${ }^{12}$.

La utilización de suturas con stapler tiene como complicaciones la filtración, hemorragia y la irreversibilidad del procedimiento, además del costo que implica el uso de la sutura mecánica. La plicatura gástrica no tendría este tipo de complicaciones. La menor agresividad de esta intervención con respecto a otras técnicas como el bypass gástrico y la manga gástrica tiene como consecuencia la no utilización rutinaria de camas de intensivos. Además esta ventaja tendría como consecuencia el menor tiempo de hospitalización post-operatoria, demostrado en los resultados preliminares ${ }^{7,12,13}$.

Recientes estudios de costo-efectividad entre la cirugía bariátrica y el tratamiento conservador en la población con obesidad mórbida, han demostrado que existe una fuerte inversión en un inicio, pero que a largo plazo la decisión quirúrgica resulta una mejor alternativa. De esta manera un tema relevante es la reducción del costo que implica este nuevo procedimiento, tanto por la no utilización de stapler, la ausencia de necesidad de camas intensivos de rutina y el menor tiempo de hospitalización. Todo lo ante- 
rior tendrá un enorme impacto en la salud pública, lo cual posiblemente producirá una re-destinación de los recursos ${ }^{17,18}$.

Si bien aún falta comprender completamente los mecanismos fisiopatológicos y tener resultados a largo plazo con esta nueva técnica, los resultados descritos en la literatura son prometedores, con una baja del exceso de peso al año similar a una gastrectomía vertical laparoscópica ${ }^{9-12}$.

\section{Referencias}

1. Global Database on Body Mass Index, an interactive surveillance tool for monitoring nutrition transition Disponible en: http://apps.who.int/bmi/index.jsp (Consultado el 9 de enero 2011).

2. Ministerio de Salud. Encuesta Nacional de Salud 20092010. Disponible en: http://www.redsalud.gov.cl/portal/ url/item/99bbf09a908d3eb8e04001011f014b49.pdf (Consultado el 17 de enero 2011).

3. Haslam DW, James WPT. Obesity. Lancet 2005;366: 1197-209.

4. Demaría Eric J. Bariatric Surgery for Morbid Obesity. N Engl J Med. 2007;356:2176-83.

5. Korner J, Leibel R. To Eat or Not to Eat - How the Gut Talks to the Brain. N Engl J Med. 2003;349:10.

6. Silecchia G, Boru C, Pecchia A, Rizzello M, Casela G, Leonetti F, et al. Effectiveness of Laparoscopic Sleeve Gastrectomy (First Stage of Biliopancreatic Diversion with Duodenal Switch) on Co-Morbidities in Super-Obese High-Risk Patients. Obesity Surgery 2006;16:1138-44.

7. Fusco P, Poggetti R, Younes R, Fontes B, Birolini D. Evaluation of Gastric Greater Curvature Invagination for Weight Loss in Rats. Obesity Surgery 2006;16:1727.

8. Fusco P, Poggetti R, Younes R, Fontes B, Birolini D.
Comparison of Anterior Gastric Wall and Greater Gastric Curvature Invaginations for Weight Loss in Rats. Obesity Surgery 2007;17:1340-5.

9. Sales C. Surset gástrico de Sales: una alternativa para cirugía bariátrica restrictiva. Rev Colomb Cir. 2008;23:131-5.

10. Talebpour M, Amoli B. Laparoscopic total gastric vertical plication in morbid obesity. J Laparoendosc Adv Surg Tech A. 2007;17:793-8.

11. Ramos A, Galvao MA, Galvao G, Evangelista L, Marins J, Ferraz Á. Laparoscopic Greater Curvature Plication: Initial Results of an Alternative Restrictive Bariatric Procedure. Obes Surg. 2010;20:913-8.

12. Brethauer S, Harris J, Kroh M, Schauer Ph. Laparoscopic gastric plication for treatment of severe obesity. Surgery for Obesity and Related Diseases (artículo en prensa).

13. Steinbrook R. Surgery for Severe Obesity. N Engl J Med. 2004;350:1075-9.

14. Kini S, Herron D, Yanagisawa R. Med Bariatric Surgery for Morbid Obesity.A Cure for Metabolic Syndrome? Clin N Am. 2007;91:1255-71.

15. Sjöström L, Narbro K, Sjöström D, Karason K, Larsson B, Wedel H, et al. Effects of Bariatric Surgery on Mortality in Swedish Obese Subjects. N Engl J Med. 2007;357:741-52.

16. Neumayer C, Ciovica R, Gadenstatter M, Erd G, Leidl S, LEH S. Significant weight loss after laparoscopic Nissen fundoplication. Surg Endosc. 2005;19:15-20.

17. Sociedad Chilena de Obesidad. Cirugía Bariátrica: ¿Una solución al problema de la Obesidad? Disponible en: http://www.sochob.cl/web/?p=833. Consultado el 15 de enero 2011.

18. Mc Ewen L, Coelho R, Baumann L, Bilik D, NotaKirby B, Herman W. The Cost, Quality of Life Impact, and Cost-Utility of Bariatric Surgery in a Managed Care Population. Obes Surg 2010;20:919-28. 